\title{
The vestibular implant: quo vadis?
}

\author{
Raymond van de Berg ${ }^{1}{ }^{*}$, Nils Guinand ${ }^{2}$, Robert J. Stokroos ${ }^{1}$, Jean-Philippe Guyot ${ }^{2}$ and Herman Kingma ${ }^{1}$ \\ 1 Department of Otorhinolaryngology and Head and Neck Surgery, Maastricht University Medical Centre, Maastricht, Netherlands \\ 2 Department of ENT, University Hospital Geneva, Geneva, Switzerland
}

\section{Edited by:}

Susan J. Herdman, Emory University, USA

\section{Reviewed by:}

Maurizio Versino, Pavia University, Italy

Giacinto Asprella-Libonati, Madonne delle Grazie Hospital ASM Matera, Italy

\section{${ }^{*}$ Correspondence}

Raymond van de Berg, Department of Otorhinolaryngology and Head and Neck Surgery, Maastricht University Medical Centre, Postbus 5800, 6202 AZ Maastricht, Netherlands. e-mail: raymond.vande.berg@ mumc.nl
Objective: To assess the progress of the development of the vestibular implant (VI) and its feasibility short-term. Data sources: A search was performed in Pubmed, Medline, and Embase. Key words used were "vestibular prosth*" and "Vl." The only search limit was language: English or Dutch. Additional sources were medical books, conference lectures and our personal experience with per-operative vestibular stimulation in patients selected for cochlear implantation. Study selection: All studies about the VI and related topics were included and evaluated by two reviewers. No study was excluded since every study investigated different aspects of the VI. Data extraction and synthesis: Data was extracted by the first author from selected reports, supplemented by additional information, medical books conference lectures. Since each study had its own point of interest with its own outcomes, it was not possible to compare data of different studies. Conclusion: To use a basic VI in humans seems feasible in the very near future. Investigations show that electric stimulation of the canal nerves induces a nystagmus which corresponds to the plane of the canal which is innervated by the stimulated nerve branch. The brain is able to adapt to a higher baseline stimulation, while still reacting on a dynamic component. The best response will be achieved by a combination of the optimal stimulus (stimulus profile, stimulus location, precompensation), complemented by central vestibular adaptation. The degree of response will probably vary between individuals, depending on pathology and their ability to adapt.

Keywords: vestibular prosthesis, vestibular implant, neural prosthesis, bilateral vestibular areflexia, bilateral vestibulopathy, adaptation, acclimation

\section{BACKGROUND AND OBJECTIVE}

The last decade, interest increases in developing an implantable vestibular prosthesis for people with a vestibular disorder, which functions analog to the cochlear implant in patients with severe sensorineural hearing loss (Gong and Merfeld, 2000, 2002; Rubinstein and Della Santina, 2002; Wall et al., 2002; Merfeld et al., 2006, 2007; Shkel and Zeng, 2006; Wall and Guyot, 2007; Gong et al., 2008; Tang et al., 2009; Fridman et al., 2010; Lewis et al., 2010; Dai et al., 2011a,b; Davidovics et al., 2011; Guyot et al., 2011a,b). The objective of this review is to assess the progress of the development of the vestibular implant (VI), its feasibility short-term, and to provide useful practical information for researchers in this field. For these purposes, the following aspects were evaluated: type of prosthesis, stimulus profile (pulse characteristics, current, frequency), stimulus site, and adaptation of the central vestibular system.

\section{MATERIALS AND METHODS DATA SOURCES}

A search was performed in Pubmed, Medline, and Embase. In order to create a very sensitive search, the key words "vestibular prosth*" and "VI" were used. The only search limit was language: English or Dutch. In order to keep up to date during writing of the review, the search was monthly reperformed until May 2011.

\section{STUDY SELECTION}

Since research of the VI is still in an experimental phase, very few studies have been published yet. Therefore all studies about the VI were included and evaluated by two reviewers. This resulted in a non-comparative and expert opinion study, not randomized controlled. No study was excluded since every study investigated different aspects of the VI.

Literature references of the selected studies were also evaluated. In case references were needed to understand the selected study, these were also selected and evaluated by the two reviewers.

\section{DATA EXTRACTION AND SYNTHESIS}

Data extraction was done by the main author. Since every study had its own point of interest with its own outcomes, it was not possible to compare data of different studies. Whenever the same research group presented data about the same subject, the data most recently obtained were used for the review.

\section{INTRODUCTION TO DATA}

Studies about the VI are sparse because its research is still in an experimental phase. Therefore, a systematic review about this subject was not an option. However, this narrative review deals with the most up to date knowledge about the most important aspects of the VI. 


\section{THE NEED FOR A VESTIBULAR IMPLANT}

There are a couple of patient groups who could benefit from the VI, since no definite therapeutic options are available for them at the moment.

The first and most important group are patients with complete or near complete acquired bilateral loss of vestibular function. This is called bilateral vestibulopathy. It leads to oscillopsia (blurred vision), chronic disequilibrium, postural instability, and impaired spatial orientation as a result of failing vestibulo-ocular and vestibulo-spinal reflexes and a reduced perception of motion and tilt (Della Santina et al., 2007; Lacour et al., 2009). Many of these patients are able to make optimal use of sensory substitution and can cope with these problems, but a substantial number of these patients show a moderate to severe reduction of their quality of life as monitored with the Dizziness Handicap Inventory (data to be published). Besides, it also increases the risk of falling, which is especially dangerous in the elderly.

Also patients with a fluctuating vestibular function such as Meniere's disease (Wall et al., 2002), elderly with presbyvertigo and patients with an incomplete centrally compensated unilateral hypofunction of a labyrinth are possible candidates for a vestibular prosthesis. (Wall et al., 2002; Agrawal et al., 2009).

No articles have been published about the selection of patients. Inclusion criteria for studies in humans in Maastricht University Medical Centre include a mean peak slow phase velocity of $\leq 5^{\circ} / \mathrm{s}$ in bilateral bithermal caloric irrigations, low or no gain at rotatory chair tests and pathological head-impulse-test (HIT) for horizontal and vertical canals in which presence of correction saccades are considered pathological (not yet published). a VI are in development and several are tested now experimentally. We are convinced however that all these technological problems will be solved in the very near future.

The output signals of the artificial labyrinth have to be converted into an adequate stimulus for the hair-cells and/or nerve. This is achieved by analog-digital conversion of the sensor outputs and feed them into a programmable digital micro-processor and signal generator. The micro-processor and signal processor enables a programmable conversion of the sensor output into analog electrical pulses to stimulate the nerves or hair-cells. In this way, a stimulus with the optimal temporal characteristics and amplitude can be delivered to the vestibular nerve or hair-cells by implanted electrodes (Gong and Merfeld, 2000, 2002; Lewis et al., 2002, 2010; Wall et al., 2002; Merfeld et al., 2006, 2007; Shkel and Zeng, 2006; Della Santina et al., 2007; Gong et al., 2008; Fridman et al., 2010; Dai et al., 2011a; Guyot et al., 2011b). A crucial problem might arise from the fact that the linear accelerometers in the artificial labyrinth like the statolith organs do not sense a difference between tilt and translation. It is yet unclear to which extend this aspect has to be dealt with within the signal processing unit of the VI, or that the brain will be able to do the necessary signal processing itself.

Optimal location of electrodes is yet to be determined (Wall et al., 2002; Wall and Guyot, 2007; Feigl et al., 2009; Guyot et al., 2011a). Monopolar as well as bipolar stimulation is possible. Bipolar stimulation is safer, provides more selective stimulation, but requires a higher current (Della Santina et al., 2007).

The prosthesis can be implanted unilaterally or bilaterally (Gong et al., 2008).

In short:

$$
\begin{aligned}
& \text { Motion input } \longrightarrow \text { Sensor } \longrightarrow \text { Processor } \longrightarrow \text { stimulus } \longrightarrow \text { Electrodes } \longrightarrow \text { Vestibular nerve } \\
& \text { - gyroscope - amplification } \\
& \text { - analog } \longrightarrow \text { digital } \\
& \text { - determining frequency } \\
& \text { - pulse generator }
\end{aligned}
$$

\section{GENERAL ASPECTS OF THE DESIGN OF THE VESTIBULAR IMPLANT}

The ultimate goal is to develop a VI which restores the vestibular function partially or completely. It directly stimulates the vestibular neural pathways by electrical pulses and has many analogies with the cochlear implant (Gong and Merfeld, 2000). However, whereas a simple microphone acts as the primary artificial sound sensor in a CI, the design of an artificial motion sensor for a VI is much more complex.

Firstly, motion (3D translations and 3D rotations) and orientation relative to the gravity vector should be detected by a combination of sensors (e.g., accelerometers, gyroscopes, Hallelements) resulting in a 6 degree of freedom (DOF) artificial labyrinth.

The major problems with the design of such an artificial labyrinth are problems with drift, limited ranges of temporal and spatial sensitivity and high power consumption. The latter might hamper an easy continuous use of an implant over time. At current, separate components or combinations of them are commercially available but still no ideal complete 6 DOF sensor fulfilling all requirements exists. New sensors specially designed to be used in
In the next chapters, the most important aspects will be discussed in more detail.

\section{PULSE CHARACTERISTICS}

Until now, the most commonly used waveform for electroneurostimulation is the charge-balanced, biphasic, rectangular, cathodic-first, current pulse (Figure 1; Gong and Merfeld, 2000, 2002; Lewis et al., 2002, 2010; Wall et al., 2002; Merfeld et al., 2006, 2007; Shkel and Zeng, 2006; Della Santina et al., 2007; Wall and Guyot, 2007; Dai et al., 2011a; Davidovics et al., 2011; Guyot et al., 2011b).

\section{CHARGE-BALANCE, MONOPHASIC, AND BIPHASIC}

With neural stimulation, chemical reversibility is a requirement. Processes occurring at an electrode, induced by a pulse, should therefore be reversed by a pulse of opposite polarity (Robblee, 1990). Monophasic and any DC-current can consequently lead to neural damage as a result of accumulated charge which induces irreversible reactions. Therefore, stimuli should be chargebalanced, which excludes the use of monophasic pulses (Shepherd et al., 1991; Shepherd and Javel, 1999; Merrill et al., 2005; 


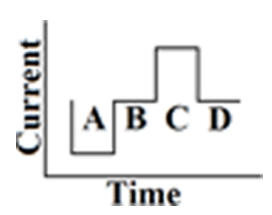

FIGURE 1 | Biphasic rectangular pulse. This means that there are two phases with charge delivery. The first phase is the cathodic (negative) one (A), after that there is a delay (B). The anodic (positive) phase follows (C), which has the same charge as the cathodic one, but with a positive charge instead of a negative. In this way the charge remains balanced. The last phase is the resting phase (D). The duration of it determines the frequency of the stimulus. A longer duration of phase $D$ implies less pulses per second (pps) and thus a lower stimulus frequency. The shape of all the phases are rectangular and a current is delivered, instead of a voltage.

Davidovics et al., 2011). A biphasic stimulus is capable of stimulating a nerve more selectively, but requires a higher current than a monophasic stimulus (Gorman and Mortimer, 1983; Shepherd and Javel, 1999).

\section{PULSE WIDTH AND AMPLITUDE}

The duration and amplitude of the pulse should be long and high enough to deliver the sufficient amount of charge to stimulate a nerve, but not too long and high to avoid damage and to limit power consumption per se. It is observed that a short high amplitude stimulus requires less charge than a wide stimulus of low amplitude in order to excite a nerve membrane (Crago et al., 1974; Mortimer, 1981; Macherey et al., 2006; Davidovics et al., 2011). However, in case of a high current, there is an increased "current spread" and "crosstalk" (see below Current).

A short pulse train with a high-frequency is also a more powerful stimulus than a long pulse train of low-frequency (Suzuki et al., 1969). In order to achieve a high-frequency, pulse width can not be too long. The optimal frequency characteristics, fall and rise time, or shape of the pulses for vestibular nerve or hair cell stimulation in humans still have to be explored in detail [see Delay Between Cathodic and Anodic Phase (Interphase Gap)].

\section{POLARITY}

The cathodic phase is the part of the pulse which induces the neural response. The anodic phase is added in order to effect balanced charge stimulation to avoid tissue damage (Brummer and Turner, 1975; Merfeld, 2004; Macherey et al., 2006; Shkel and Zeng, 2006). The anodic phase is also capable of eliciting a response, but it is less efficient and requires higher currents. During tests in bilaterally plugged squirrel monkeys it was shown the cathodic phase is the determining phase (Gong et al., 2008). Still, this does not mean that in all patients a biphasic-cathodic-first stimulus should be used.

After all, central processes are shown to be more sensitive to anodic stimuli than to cathodic ones, while for intact peripheral axons it is just the opposite (Rattay et al., 2001; Macherey et al., 2006). When many peripheral axons have been degraded, an anodic stimulus could therefore be more efficient than a cathodic (Macherey et al., 2006). It is important to understand the pathology of a patient to know whether peripheral axons have degraded. This could imply a different stimulus profile for different pathologies. During investigations on polarity in

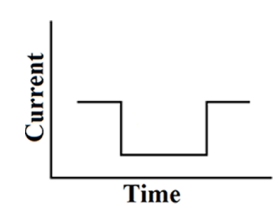

FIGURE 2 | Monophasic pulse.

cochlear implants, there was often no difference between anodic and cathodic stimuli, although sometimes a cathodic stimulus created a better response. This "central activation hypothesis" is therefore still questioned (Macherey et al., 2006), but remains a factor to take into consideration.

\section{DELAY BETWEEN CATHODIC AND ANODIC PHASE (INTERPHASE GAP)}

The delay between the cathodic and anodic phase is a delicate balance. It should be long enough to prevent slow action potentials from the cathodic phase to be inhibited by the anodic phase (Macherey et al., 2006; Della Santina et al., 2007; Gong et al., 2008). However, it should not be too long, otherwise the anodic phase will generate action potentials instead of balancing the charge (Della Santina et al., 2007). Also, it should be as short as possible in order to reduce the damage to electrodes (Gong and Merfeld, 2002). How short the delay can be, is not yet known (Gong et al., 2008). In chinchillas, varying interphase gap has not shown a discernible effect on VOR response or axis misalignment (Davidovics et al., 2011).

\section{OTHER WAVEFORMS THAN RECTANGULAR}

Next to rectangular, many different waveforms have been described and applied (Balter et al., 2004; Macherey et al., 2006). Rise and fall time and pulse shape are significant factors in frequency characteristics, stimulus efficiency, and reducing power consumption, while maintaining charge-balance (Macherey et al., 2006). Most of these waveforms are only tested in cochlear implants and not (yet) with a VI. The most well-known and eligible waveforms are now discussed.

\section{Monophasic}

A single cathodic or anodic pulse (Figure 2). As indicated already above, since charge-balance is not maintained, this is an obsolete waveform for stimulating vestibular afferents.

\section{Pseudomonophasic}

A short phase of polarity, directly followed by a longer phase with lower amplitude of opposite polarity (Figure 3). In cochlear implants, pseudomonophasic stimulation has shown to be more efficient by producing lower thresholds than a biphasic rectangular waveform. It also reduces spread of excitation (Frijns et al., 1996; Macherey et al., 2006).

\section{Delayed pseudomonophasic}

A customized pseudomonophasic waveform, where the longer phase is presented midway between the short phases of two consecutive phases (Figure 4). As a result of the delay between the short and long phase, its stimulation efficiency in cochlear implants is 


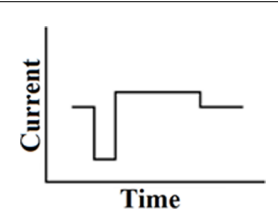

FIGURE 3 | Pseudomonophasic pulse.

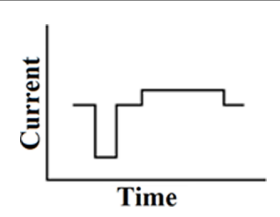

FIGURE 4 | Delayed pseudomonophasic pulse.

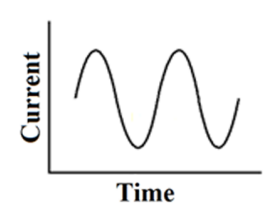

FIGURE 5 | Continuous 1-co-sinusoidal stimulation.

even better than a pseudomonophasic waveform. This results in a reduction of power consumption. The first phase is the dominant phase. Lengthening or lowering of the second phase does not change the produced thresholds. A delayed pseudomonophasic is not capable of producing frequencies as high as a biphasic rectangular waveform, as a result of the prolonged second phase (Macherey et al., 2006).

\section{Continuous 1-co-sinusoidal stimulation}

This waveform has been tested with galvanic vestibular stimulation via large surface electrodes placed on the skin retro-auriculary. It produced the most reproducible galvanic induced body sway at around $0.5 \mathrm{~Hz}$. It was compared with low-frequency block pulses, trapezoidal pulses, short pulses, and 1-co-sinusoidal pulses (Balter et al., 2004). This stimulus is currently used to explore electrical excitability in patients with bilateral vestibulopathy, but clearly differs from the frequency range applied for direct stimulation of the labyrinth or nerve. It has to be noted that the body sway frequency response also depends on specific mechanical properties of the body (resonance frequency, mass inertia, etc.; Figure 5).

\section{CURRENT}

Voltage sources are simpler and more efficient than current sources. However, in contrary to current sources, they are not able to control the amount of charge delivery when impedance of electrodes varies. Therefore, a current source is the selected stimulation modality (Wall et al., 2002).

The amplitude of the current has yet to be determined. In nature, physiologically, the conversion from the analog hair cell receptor potential via synapses into spike trains in the afferent fibers can be considered as comparable to a process of analog to digital conversion. Here the sensory information in the spike train is coded in terms of frequency and phase of spikes. The amplitude of the spikes seems then of limited importance: it will show an on-off characteristic. This implies that maybe also the amplitude of the artificial electrical nerve fiber stimulation only needs to pass a minimum threshold to be effective. No clear relationship then would exist between pulse amplitude and frequency and phase content of the original sensor signal. However, the amplitude of the electrode pulses determines also the current spread and by that the number of fibers that will be excited with possibly different thresholds (irregular and regular units) per fiber. It is well-known that synchronization of activity between fibers play an important role in the ultimate response. So increasing amplitude will activate more fibers and initiate synchronization.

There are three key factors which play a role in the pulse amplitude: "safety," a "sufficient response" and "current spread, synchronization, and crosstalk." Regarding safety, the amplitude of the current must not be too high $(>40 \mathrm{mC})$, otherwise it will cause electrode dissolution and neural damage (Gong and Merfeld, 2000; Wall et al., 2002; Davidovics et al., 2011). However, if the current is too low, only the irregular afferents will be stimulated and there will not be a sufficient response. Most units are regular and they require a higher current (Bronte-Stewart and Lisberger, 1994; Fridman et al., 2010). Investigations have shown that when the amplitude of current increases, magnitude of VOR also increases, which leads to a higher gain and a more sufficient response (Bronte-Stewart and Lisberger, 1994; Gong and Merfeld, 2000; Della Santina et al., 2007; Merfeld et al., 2007; Wall and Guyot, 2007; Gong et al., 2008; Fridman et al., 2010). When current becomes too high again, the electricity will be spread among other than the targeted anatomical structures ("current spread") and they will be stimulated and react accordingly ("crosstalk"). With the VI, crosstalk mainly appears in the facial nerve, cochlear nerve, and the other ampullary nerves (Lewis et al., 2002; Merfeld et al., 2006; Shkel and Zeng, 2006; Della Santina et al., 2007; Wall and Guyot, 2007; Fridman et al., 2010; Davidovics et al., 2011). This limits selective stimulation (Fridman et al., 2010). In order to find a way of avoiding crosstalk from the facial nerve while having the most optimal current, the amplitude has been increased until facial twitching was observed. After that, it was decreased with a few microampere until no twitching was present anymore. This experiment was done in squirrel monkeys and chinchillas, without observing interference with the cochlear nerve (Merfeld et al., 2007; Fridman et al., 2010). To avoid crosstalk with the other ampullary nerves, different strategies have been proposed which can be used simultaneously: decreasing current, electrode position and isolation, current steering, and precompensation. The first two speak for themselves: by decreasing the current until no or acceptable shift from the intended VOR-axis is obtained (which means that there is no or little stimulation of the other ampullary nerves) or by locating a well isolated electrode as near as possible to the ampullary nerve so little current is necessary to adequately stimulate the ampullary nerve, crosstalk can be limited (Della Santina et al., 2007; Merfeld et al., 2007; Wall and Guyot, 2007; Feigl et al., 2009). Current steering is the use of stimulating multiple electrodes at the same time, in order 
to steer the current to a location that is in between the targeted area's of the electrodes when all the stimulating current is delivered to either one of them. The center of activity can therefore be shifted by changing the delivered current to an electrode (Bonham and Litvak, 2008). This is already investigated in cochlear implants, but not yet with the VI. With precompensation, current spread from an electrode targeting one ampullary nerve, is overcome by adjusting the input of the electrodes targeting the other ampullary nerves using vector summation. By using vector summation, it is possible to significantly restore the VOR-axis and reduce errors in the speed of eye movement responses (obtaining a bigger amplitude). This process of accurately correcting the misalignment of VOR-axis, is called precompensation. Whether precompensation is needed in human subjects is still unknown, since current spread to other ampullary nerves is believed to be less due to bigger distances between anatomical structures in humans compared to monkeys and chinchillas (Merfeld et al., 2007; Fridman et al., 2010). Besides, neuroplasticity is also able to counterbalance effects of current spread to an extend (Merfeld et al., 2006, 2007). Also adaptation ability to adjust the 3D VOR has shown to be impressive: the human horizontal VOR can be inverted completely within several days when wearing glasses with inverted prisms (Melvill Jones et al., 1988). This will be discussed subsequently.

\section{PULSE FREOUENCY}

Firstly, increase of pulse frequency induces an increase of VOR magnitude (Cohen et al., 1965; Gong and Merfeld, 2000; Wall et al., 2002; Wall and Guyot, 2007), mimicking the natural response. Secondly, pulsatile stimulation composed of high-frequency components yields less current spread than low-frequency stimulation (Rubinstein and Spelman, 1988; Merfeld et al., 2006). Therefore, the stimulation frequency should be a high pulsatile stimulation which can be varied along the whole frequency range of the afferent fiber (0-400 pps). Whether there is a maximum of stimulation frequency below the natural range is uncertain. Some data suggest that there is a maximum around 200 pps for the posterior ampullary nerve (PAN) in humans (Wall and Guyot, 2007), although this limitation was not encountered in other experiments in animals (Merfeld et al., 2007; Fridman et al., 2010; Lewis et al., 2010; Davidovics et al., 2011).

A third important fact is the baseline firing rate of vestibular afferents not being centered in the middle of its frequency range (Gong and Merfeld, 2000, 2002; Shkel and Zeng, 2006). As a result, the dynamic range below the baseline is much less than above. In other words: there is less space to maneuver below baseline than above. This is not a problem with a bilateral implant, because the "push-pull" effect is used (Gong and Merfeld, 2002; Della Santina et al., 2007; Gong et al., 2008). However, a unilateral implant is probably not able to give adequate information about yaw-rotations in contralateral direction because the dynamic range below baseline is too small. Since a unilateral implant is preferred because it requires less surgery, risks, costs, etcetera, it could be worth solving this problem. Therefore, the baseline can artificially be set at a higher frequency (supranormal frequency) than the normal average firing rate at a frequency between baseline and maximum. It provides a broader range to modulate below baseline and could therefore be sufficient to provide adequate bidirectional vestibular sensations with a unilateral prosthesis (Gong and Merfeld, 2000, 2002; Lewis et al., 2002, 2010; Della Santina et al., 2007; Merfeld et al., 2007). Investigations show that it is possible to increase the baseline firing rate and that the nervous system is able to adapt. At first a brisk nystagmus occurs after setting a new baseline, which decreases over time while it is still possible to create a response by modulating above and below the baseline. The fact that a brisk contralateral nystagmus occurs when the supranormal baseline stimulation stops, suggests that central compensation is able to accept a new baseline (Gong and Merfeld, 2002; Lewis et al., 2002, 2010; Merfeld et al., 2006, 2007). Others believe that it is not necessary to use supranormal baseline frequencies because the asymmetry is usually well compensated by the adaptive capacity of vestibulocerebellar circuits. It is enough to suffer little of disability when having a single normal labyrinth (Curthoys and Halmagyi, 1995; Black et al., 1996; Fridman et al., 2010). Besides, modulating around a supranormal baseline frequency provides the vestibular system with information that differs markedly from that encountered in everyday life. This could lead to slower and less complete adaptation (Dai et al., 2011a). Therefore, in their opinion, the advantages of a supranormal frequency (broad dynamic range, symmetric responses) do not counterbalance the major advantage of a normal firing rate, which is higher gain and more sufficient adaptation (Fridman et al., 2010; Dai et al., 2011a). It is indeed observed that static compensation is quite good, but in contrast, dynamic compensation remains poor: e.g., the HIT (image stabilization) remains abnormal even after complete central compensation and adaptation. Also, many patients report a reduced automatization of balance, orientation, and navigation even in centrally good compensated unilateral vestibular lesions (Lacour et al., 2009).

The fourth fact is that when modulating around the baseline, an adequate modulation sensitivity should be chosen. Modulation sensitivity is the amount of modulation in firing frequency as a function of angular head velocity. In other words: for each degree per second that a head turns, the firing rate is modulated up- or downward in pulses per second (pps). The amount of pulses per second increase of firing rate for a given angular velocity is called the sensitivity. Sensitivity is therefore noted as pps ${ }^{\circ}$ \%s (Gong and Merfeld, 2000; Lewis et al., 2002; Merfeld et al., 2007). The firing frequency of vestibular neurons forms a hyperbolic curve: it is linear for a broad range of angular velocities, only at higher velocities the response saturates and sensitivity is lower. Therefore, a wide range of velocities can be covered with a fixed frequency range and a fixed sensitivity (Gong and Merfeld, 2000, 2002; Merfeld et al., 2007). In the natural system, type I neurons have a baseline firing rate of $90 \mathrm{pps}$, with a modulation sensitivity of $0.5 \mathrm{pps} / \%$ s (Goldberg and Fernandez, 1971; Guyot et al., 2011a). Thus, an increase $0-20 \%$ in head velocity results in an increase from 90 to 100 pps. It is possible to artificially increase the sensitivity (Gong and Merfeld, 2000, 2002; Lewis et al., 2002, 2010; Merfeld et al., 2007). This results in a significantly higher gain but lower range and implies that less angular velocities can be covered as maximum stimulation is then achieved sooner at slower velocities (Merfeld et al., 2007; Lewis et al., 2010). 


\section{STIMULUS SITE}

The optimal stimulus location in humans has not been determined yet, because there are three difficult basic requirements. Firstly, when stimulating at a certain position, it should be selective and give as less crosstalk as possible. This is important, because stimulation in the vestibular area can interfere with hearing and stimulate the facial nerve and other vestibular branches than the intended one (Wall et al., 2002; Merfeld et al., 2006, 2007; Della Santina et al., 2007; Wall and Guyot, 2007; Feigl et al., 2009; Guyot et al., 2011b). This can be solved by inserting the electrode as close as possible to the vestibular nerve (Merfeld et al., 2006; Della Santina et al., 2007; Wall and Guyot, 2007; Feigl et al., 2009; Guyot et al., 2011a,b). However, it could interfere with the second basic requirement: as few surgical risks as possible. Major complications of some surgical techniques are deafening the patient and damage to the facial nerve (Gacek, 1974; Parnes and McClure, 1990, 1991; Wall and Guyot, 2007; Feigl et al., 2009; Tang et al., 2009; Dai et al., 2011b). To prevent the hearing loss, experiments in humans are still done with deaf patients (Wall and Guyot, 2007; Guyot et al., 2011a,b). Since the ultimate goal is to develop an implant which can also be used in patients with only a vestibular problem, it is important to refine surgical techniques and eventually weigh the pros of reducing crosstalk against its contras which are the complications involved.

Thirdly, it is still uncertain which part of the nerve should be stimulated. It could depend on pathology since different parts of the vestibular sensory system are affected to a different extend. Gradual and partial loss (e.g., presbyvertigo) seem to implicate slow transganglionic degeneration of vestibular fibers with lower postural deficits and faster compensation than a sudden and total loss (e.g., neurectomy) which leads to fast Wallerian degeneration and needs deep reorganization of the neuronal networks to recover (Lacour et al., 2009).

Therefore, it is necessary to understand the pathological processes of diseases, in order to determine electrode position (Wall et al., 2002; Lacour et al., 2009).

Studies show that loss of vestibular hair-cells leads to some degeneration of vestibular nerve and ganglion cells (Schuknecht, 1982; Cass et al., 1989), while a significant number of surviving cells still responds to electrical stimulation, without change of threshold (Wall et al., 2002). This information has to be combined with studies about the specific diseases. Presbyvertigo is associated with an age-related reduced elasticity of the cupula and statolith membrane, a change in viscosity of the endolymph, linear loss of hair-cells, nerve fibers, ganglion cells, and the degeneration of within the cerebellum and vestibular nuclei. The mechanical changes in the labyrinth (elasticity and viscosity) reduce the sensitivity, especially for the higher frequencies (fast head movements). In the cristae, type II cells are lost at a greater rate than in the maculae, in contrary to type I cells which have an equal loss in the cristae and maculae. Total reduction of sensory hair-cell population averages $20 \%$ for the maculae and $40 \%$ for the cristae, with pronounced inter-individual variations. Reduction in number of vestibular nerve fibers averages $37 \%$ in individuals between 75 and 85 years compared to younger persons and the vestibular nuclei show a neuronal loss with an average of 3-5\% per decade between the ages of 40 and 93 years (Lacour et al., 2009). The superior division of Scarpa is significantly more affected than the inferior division (Velazquez-Villasenor et al., 2000; Rauch et al., 2001). Temporal bone studies on patients with Menière's disease show next to presbyvertigo degeneration, also a significant loss of hair-cells and ganglion cells, with the utricular macula being relatively spared (Leake et al., 1999; McCall et al., 2009). Regarding ototoxicity, degeneration depends on type of drug, drug dosage, and duration of treatment. However, especially hair-cells seem to degenerate without affecting ganglion cells on the short-term (Tsuji et al., 2000a; Lacour et al., 2009).

These differences in survival and plasticity of neurons lead to different amounts of neurons available for the electrostimulation. This will result in a wide variation of inter-individual responses to vestibular prosthetic stimulation (Fridman et al., 2010).

Taken all these data into account, it is suggested that after hair cell insult, peripheral dendrites will initially remain intact, after which they "die back" to Scarpa's ganglion (Wall et al., 2002). Electrically stimulating the vestibular nerve could probably prevent rapid loss of spiral ganglion nerves (Leake et al., 1999; Shepherd et al., 2008). Therefore, depending on pathology, direct stimulation after a vestibular insult at different stimulation sites should be considered (Wall et al., 2002). Main sites are locations along the course of the vestibular branches or Scarpa's ganglion (Gacek, 1974; Parnes and McClure, 1990, 1991; Kudo and Nomura, 1996; Gong and Merfeld, 2000, 2002; Lewis et al., 2002, 2010; Wall et al., 2002; Merfeld et al., 2006, 2007; Della Santina et al., 2007; Wall and Guyot, 2007; Feigl et al., 2009; Fridman et al., 2010; Guyot et al., 2011a). The most commonly used surgical approaches will now be discussed briefly, as well as some proposed techniques.

\section{APPROACH TO THE CANALS/AMPULLOTOMY}

Most investigations use an approach to the ampullae in which the facial nerve and middle ear structures are spared. The surgical procedure involves a cortical mastoidectomy after which the semicircular canals are exposed and opened. Depending on the study, a fenestration at the thin segment or near the junction of the thin segment and the ampulla is made and the electrodes are inserted and placed near the crista of the ampulla.

Animal studies confirm that an electrically evoked nystagmus can be induced, which corresponds to the plane of the stimulated canal (Gong and Merfeld, 2000, 2002; Lewis et al., 2002, 2010; Rubinstein and Della Santina, 2002; Merfeld et al., 2006, 2007; Gong et al., 2008; Tang et al., 2009; Fridman et al., 2010; Davidovics et al., 2011).

However, there are two major risks. Firstly, as mentioned earlier, stimulating the ampullae could lead to an insufficient response since the ampullae can be too far away from a vital part of the vestibular nerve (Schuknecht, 1982; Cass et al., 1989; Leake et al., 1999; Wall et al., 2002; Lacour et al., 2009). Secondly, there could be interference with hearing. This can be the result of opening the labyrinth or by activating the cochlear nerve due to current spread of the electrodes. A study in rhesus monkeys shows that implantation can lead to a hearing loss but is not likely to be more than $10 \mathrm{~dB}$. This hearing loss has not yet been proven to be significant. Regarding stimulation of the cochlear nerve, levels of ABR (auditory brain stem response) and DPOAE (distortion product otoacoustic emissions) increased during stimulation with 
$0-5$ and $2-14 \mathrm{~dB}$ respectively (statistically not significant; Dai et al., 2011b).

Research in Maastricht University Medical Centre has focused on modifying this technique to a well-defined ampullar approach which has proven to generate an electrically evoked nystagmus in the plane of the stimulated canal in a human (not yet published).

\section{APPROACH TO THE POSTERIOR AMPULLARY NERVE BY GACEK, MODIFIED BY GUYOT ET AL. (2011a,b)}

Gacek (1974) described the surgical approach to the PAN, in order to treat patients with disabling benign paroxysmal positional vertigo. By modifying this technique, it is possible to achieve a robust vertical nystagmus which corresponds to a large extend to an axis perpendicular to the plane of the posterior semicircular canal, without any apparent crosstalk (Wall and Guyot, 2007). A transmeatal approach is used, in which the lateral bony ridge overhanging the round window niche is removed to expose the whole membrane of the round window. The canal of the PAN is then approached by drilling in the most rostral part of the floor of the round window niche. Next, it is "blue-lined" by leaving approximately $100 \mu \mathrm{m}$ of bone on it. After that, an electrode is inserted. This approach has been successfully tested in humans (Wall and Guyot, 2007).

The major risk of this technique is sensorineural hearing loss, which varies from 3.7 (Gacek and Gacek, 2002) to 38\% (Epley, 1980).

\section{APPROACH OF THE LATERAL AMPULLARY NERVE AND SUPERIOR AMPULLARY NERVE BY GUYOT ET AL. (2011a,b)}

A new technique was proposed by Guyot et al. (2011a,b) in order to reach the lateral ampullary nerve (LAN) and superior ampullary nerve (SAN) with little risk of damage to the facial nerve and without affecting the oval window. It is a transmeatal approach with a partial atticotomy in which the the head of the malleus and the incus are removed. The LAN and SAN are then approached by drilling at the spot ventral to the prominence of the lateral semicircular canal, superior to the horizontal tympanic segment of the facial canal and inferior to the tegmental roof. The drill is directed ventrally, medially, and inferiorly. Once the nerves are reached, electrodes can be inserted. A temporal bone study showed that the canal of the nerve was directly reached in few cases (5-12.5\%). Mostly, it was indirectly accessible through the osseous ampulla by removing parts of the bony wall of the lateral semicircular canal, but with preservation of the membranous labyrinth (70-90\%).

This technique has recently been tested in three humans. It is shown that a nystagmus is evoked by stimulating the nerves at the desired location. When LAN is separately stimulated, a horizontal nystagmus is obtained. However, in two out of three cases, LAN and SAN were simultaneously stimulated since their fiber bundles are in close proximity of each other (Wall et al., 2002; Della Santina et al., 2007; Guyot et al., 2011a). This resulted in a predominantly horizontal nystagmus with a vertical component. Being not able to selectively stimulate the nerves might not be a problem, since cross-axis adaptation would possibly align the nystagmus to the desired axis. Stimulation range varied between 120 and $1000 \mu \mathrm{A}$.

Regarding risks, sensorineural hearing loss and damage to the facial nerve will probably be the main risk of this technique because the membranous labyrinth can be damaged accidentally and drilling is performed closely to the facial nerve (Feigl et al., 2009). Also, the ossicular chain has to be reconstructed as parts of the chain are removed during the procedure. This results in an air-bone gap, which can be expected to be less than $20 \mathrm{~dB}$ in more than $90 \%$ of cases (Zheng et al., 1996; Guyot et al., 2011a).

\section{APPROACHES TO SCARPA'S GANGLION}

Since the spatial orientation of the five vestibular receptors is preserved in Scarpa's ganglion (Sando et al., 1972), it could be possible to implant electrodes at that location and provide adequate stimulation. However, current spread could be an issue because the fiber bundles are (just like LAN and SAN) in close proximity of each other. It would also require very invasive approaches to get the electrodes in position: a translabyrinthine or middle fossa approach (Wall et al., 2002). These are not yet investigated regarding the VI and will therefore not be discussed. Since in many pathological processes a significant number of vestibular nerve cells survive (Schuknecht, 1982; Cass et al., 1989; Tsuji et al., 2000a,2000b; Velazquez-Villasenor et al., 2000; Rauch et al., 2001; Lacour et al., 2009) and approaches to Scarpa's ganglion are very invasive, research has only been done about approaches to the ampullae and vestibular nerves (Suzuki et al., 1969; Kudo and Nomura, 1996; Gong and Merfeld, 2000; Lewis et al., 2002, 2010; Merfeld et al., 2006, 2007; Wall and Guyot, 2007; Gong et al., 2008; Feigl et al., 2009; Tang et al., 2009; Fridman et al., 2010; Dai et al., 2011b; Guyot et al., 2011a).

Until now, most research has focused on stimulating the nerves of the semicircular canals and not the ones of the otolith organs. This results from the fact that the sensory part of the otolith organs, the macula, consists of hair-cells which are sensitive for motion in different directions (Wall et al., 2002; Kingma, 2005; Lysakowski, 2005). This means that their nerves have axons which represent different directions. Therefore, they are difficult to stimulate. It would result in much crosstalk and require extra electrodes and accelerometers (Wall et al., 2002; Della Santina et al., 2007). Some state that it is also much more important to emulate the angular VOR from the semicircular canals, than the linear VOR from the otolith organs. After all, when one is fixating at a target greater than $\sim 1 \mathrm{~m}$ away, retinal image slip due to angular motion of the eyes and head dominate slip due to translation (Della Santina et al., 2007; Fridman et al., 2010).

Although the brain is able to adapt to a suboptimal stimulus (see below), striving for an optimal stimulus seems important in order to reduce the burden on central compensation (Fridman et al., 2010). Finding the best stimulus site is an important step in this process.

\section{RESULTS}

Studies have shown that electric stimulation of the canal nerves induces a nystagmus which corresponds to the plane of the canal which is innervated by the stimulated nerve branch (Gong and Merfeld, 2000, 2002; Lewis et al., 2002, 2010; Wall et al., 2002; Della Santina et al., 2007; Merfeld et al., 2007; Wall and Guyot, 2007; Gong et al., 2008; Fridman et al., 2010; Guyot et al., 2011a). The outcomes still demonstrate inter-subject variability, just like with 
cochlear implant patient outcomes. However, there are many consistencies (Merfeld et al., 2007). The characteristics of the evoked VOR will now be discussed. Main results are presented in Table A1 in Appendix.

\section{GAIN}

The gain of the electrically evoked VOR varies from very low (e.g., 0.07 ) to near normal. It is influenced by many factors. Apart from the efficacy of the electrode-nerve system, there are factors that can be modified more easy in order to achieve a higher gain.

\section{Current}

As stated earlier, gain is increased by a higher current (BronteStewart and Lisberger, 1994; Gong and Merfeld, 2000; Wall et al., 2002; Della Santina et al., 2007; Merfeld et al., 2007; Wall and Guyot, 2007; Gong et al., 2008; Fridman et al., 2010). Current should be high enough to stimulate the regular units of the nerve, but low enough to avoid electrode dissolution, neural damage, and too much crosstalk by current spread (Gong and Merfeld, 2000; Lewis et al., 2002; Wall et al., 2002; Merfeld et al., 2006, 2007; Shkel and Zeng, 2006; Della Santina et al., 2007; Wall and Guyot, 2007; Fridman et al., 2010; Davidovics et al., 2011). Methods to overcome these factors, like electrode position and isolation, current steering, and precompensation are still investigated (Suzuki et al., 1969; Kudo and Nomura, 1996; Gong and Merfeld, 2000; Wall et al., 2002; Della Santina et al., 2007; Merfeld et al., 2007; Wall and Guyot, 2007; Bonham and Litvak, 2008; Feigl et al., 2009; Fridman et al., 2010; Guyot et al., 2011a).

\section{Frequency}

Gain increases with stimulus frequency. A frequency range has not be determined yet, since it varies with experimental set-up and tested subject (Cohen et al., 1965; Gong and Merfeld, 2000; Wall et al., 2002; Wall and Guyot, 2007). However, it might be necessary to increase the dynamic range, which implies an increase of the baseline firing rate of vestibular afferents to a supranormal level (Gong and Merfeld, 2000, 2002; Lewis et al., 2002, 2010; Della Santina et al., 2007; Merfeld et al., 2007). As discussed above, this might however result in unacceptable lower gains (Fridman et al., 2010).

\section{Modulation sensitivity}

A significantly higher gain is achieved by adjusting the settings of the vestibular prosthesis to a higher modulation sensitivity (Gong and Merfeld, 2002; Lewis et al., 2002, 2010; Merfeld et al., 2007). It can result in near normal gain and for each doubling of stimulation sensitivity, gain roughly doubles (Merfeld et al., 2007).

\section{Stimulation period and transitions}

Gain is significantly increased by stimulation period and transitions between stimulation states. When stimulating for a longer time, gain at first decreases, but slowly increases over the ensuing time (Lewis et al., 2002, 2010). Considering transitions between different stimulation states (investigated transition cycle: off - low sensitivity $\rightarrow$ low sensitivity - high sensitivity $\rightarrow$ high sensitivity - off $\rightarrow$ off - low sensitivity, etc.), it is shown that the gain at the onset of stimulation with a new off-to-on transition is generally greater than that recorded after the previous off-to-on transition. With each new cycle, gain initially increases followed by a rapid drop. After that, it either slowly increases or remains relatively stable at a level which is above the one of the previous cycle. Which transition is most important in increasing the gain, is not yet determined (Lewis et al., 2010).

\section{Bilateral stimulation}

Bilateral stimulation increases the gain. The electrically evoked responses by bilateral stimulation turn out to be equal to a linear summation of responses evoked by unilateral right ear and unilateral left ear stimulation. Advantages of bilateral stimulation are a higher sensitivity (more precise stimulation) and the fact that it is probably not necessary anymore to use a supranormal baseline firing rate. Disadvantages are additional risks (e.g., perform surgery on two sides), higher costs, and more complexity. After all, the efficacy of the electrode-nerve system is hardly ever equal on both sides, which requires different currents on each side in order to restore the push-pull nature of the complementary functioning labyrinths (Gong and Merfeld, 2002; Della Santina et al., 2007; Gong et al., 2008).

\section{Age}

VOR-gain declines with advancing age (Dimitri and Oas, 1996; Lewis et al., 2010). However, there seems to be no good parallel between imbalance in the elderly and caloric response. This could imply that age-related decline in vestibular response is the result of a decline in central vestibular processing instead of a loss of peripheral vestibular function, or that VOR-gain is only affected in the higher frequencies (Mallinson and Longridge, 2004).

\section{ADAPTATION TO BASELINE}

When the vestibular afferents are chronically stimulated by a tonic continuous (supra)normal baseline pulse-rate, at first a brisk nystagmus occurs. This nystagmus decreases, dependent on the laboratory animal setting, over a period from $20 \mathrm{~min}$ to 1 day until no nystagmus is recorded anymore (Gong and Merfeld, 2000; Lewis et al., 2002, 2010; Merfeld et al., 2006, 2007; Della Santina et al., 2007; Gong et al., 2008; Guyot et al., 2011b). During PAN stimulation in a human with repeated "on-off" transitions, nystagmic response disappeared after only a few minutes without major discomfort (Guyot et al., 2011b). When stimulation is stopped, a nystagmus in the contralateral direction occurs, which attenuates within minutes to hours. This "after-effect" suggests a form of neural plasticity and adaptation (Merfeld et al., 2006, 2007; Guyot et al., 2011b). The fact that this "after-effect" has a more rapid decay, is suggested to be the result of the "recall" of the previous adaptive state (no stimulation; Gong and Merfeld, 2002).

\section{MOTION-MODULATED RESPONSES}

Although the nervous system adapts to the tonic baseline firing rate, it does not adapt to the motion-modulated part of stimulation. VOR develops significantly when the stimulation frequency is modulated around the baseline. When stimulated for a couple of months, motion-modulated responses still remain. Gain is initially high and declines rapidly, after which it slowly increases (Lewis et al., 2002, 2010; Merfeld et al., 2006, 2007; Gong et al., 2008; Guyot et al., 2011b). 


\section{DUAL STATE ADAPTATION}

Transitions between stimulation states do not only have a favorable effect on gain, but also on the evoked nystagmus when the prosthesis is activated or inactivated. After a couple off-to-on and on-to-off and transitions, nystagmus, and the "after-effect nystagmus" are less intense and the return to baseline occurs more rapidly. After many of these transitions, only little nystagmus is evoked when stimulation starts or stops. The nervous system of laboratory animals seems to recognize the absence or presence of stimulation and react adequately to it. This adaptation to two states ("on" or "off") in which no major undesired nystagmus response is evoked by a transition, is called "dual state adaptation." This might be relevant for patients who only need the prosthesis on a stand-by system to overrule fluctuations (e.g., Meniere's disease), or when a change of batteries or stimulation parameters is necessary (Merfeld et al., 2006, 2007; Lewis et al., 2010; Guyot et al., 2011b).

\section{CROSS-AXIS ADAPTATION}

It is possible to develop an eye response which is aligned with the axis of head motion, by stimulating a canal which is orthogonal to the motion axis. This is called "cross-axis adaptation" (Lewis et al., 2002; Della Santina et al., 2007; Dai et al., 2011a). In an experiment with squirrel monkeys, the PAN was stimulated while the velocity sensor of the prosthesis was oriented parallel to the axis of the lateral canals. At first a vertical VOR of 0.05 was measured, as well as a small horizontal response. Over 7 days, the horizontal gain increased to 0.1 and the vertical gain decreased (Lewis et al., 2002). This indicates that cross-axis adaptation is possible. It could play a role in correcting misalignments of the device (Della Santina et al., 2007; Dai et al., 2011a).

\section{TIME CONSTANT}

The time constant of the evoked VOR is smaller than the time constant of the prosthesis and does not change significantly during chronic stimulation, bilateral stimulation, and by multiple transitions in modulation sensitivity (Merfeld et al., 2007; Gong et al., 2008; Lewis et al., 2010). Probable underlying mechanisms are that the velocity storage integrator is not engaged by prosthetic input and the high levels of tonic stimulation which are likely to reduce the efficacy of synaptic transmission in the central vestibular pathways by constantly releasing synaptic vesicles (Lewis et al., 2010). Since the time constant of the prosthesis can be made arbitrarily long, it has been suggested that the smaller time constant of the evoked VOR might not be a limitation (Merfeld et al., 2007). However, much depends also to which extend involvement of the velocity storage is crucial for further central processing.

\section{VOR-SYMMETRY}

When stimulation starts, a substantial VOR-asymmetry is present. Head turns away from the stimulated ear produce larger VORgains than head turns toward the stimulated ear. This phenomenon was observed in squirrel monkeys, which also showed a decline in difference between the ipsilateral and contralateral gains of 71$78 \%$, when stimulated chronically for 2 weeks in low sensitivity mode [0.9-1.2 pps $/ \%$ s (Lewis et al., 2010)]. In another experiment with chinchilla's, VOR-asymmetry did not change significantly during 1 week of prosthetic use. Improvement in VOR-symmetry when using a prosthesis is therefore still uncertain (Dai et al., 2011a).

\section{VOR-AXIS}

When the prosthesis is activated for the first time, there is a rapid deviation of the intended VOR-axis. This results from current spread and reduction of the intended VOR-gain (e.g., reduction of horizontal VOR-gain when the horizontal semicircular canal is stimulated; Fridman et al., 2010; Lewis et al., 2010).

In chinchillas it is shown that VOR-axis remains nearly constant when modulating the intensity of frequency from 20 to $100 \%$, but misalignment increases with increasing current amplitude and pulse duration. Therefore, the response of an electrode which elicits suboptimal selective stimulation and moderate to severe misalignment (probably because it was implanted not very close to the ampullary nerve), can be optimized by using a lower pulse duration. However, an electrode eliciting highly selective stimulation, maintains low misalignment over a wide range of pulse duration (Davidovics et al., 2011).

During chronic stimulation, the VOR-axis shifts back toward the intended axis (Lewis et al., 2010; Dai et al., 2011a). In another experiment with chinchillas, misalignment of VOR-axis improved significantly after 1 week of multichannel prosthetic stimulation. This indicates a rapid adaptation of the central nervous system to prosthetic stimulation (Dai et al., 2011a).

As stated earlier, precompensation might be able to significantly correct the misalignment of VOR-axis. Also, modulating around a normal baseline instead of a supranormal one could lead to faster and more complete adaptation (Fridman et al., 2010; Dai et al., 2011a).

\section{ACCLIMATION TO PERIPHERAL VESTIBULAR STIMULATION BY A PROSTHESIS}

Still little is known about the ability of the brain to adapt to changes in the peripheral vestibular signal (Lewis et al., 2002). Particularly Merfeld and colleagues have done extensive research to adaptation in guinea pigs and squirrel monkeys (Gong and Merfeld, 2000, 2002; Lewis et al., 2002, 2010; Wall et al., 2002; Merfeld et al., 2006, 2007; Gong et al., 2008).

Investigations show that the brain is at least partly able to adapt to changes in peripheral vestibular signal by a prosthesis. Examples are: a significant increase in gain as a result of chronic stimulation and multiple transitions between stimulation states, the "after-effect" and "recall," "dual state adaptation," "cross-axis adaptation" and an increase of VOR-symmetry, and a better alignment of VOR-axis during chronic stimulation.

Summarizing: the brain is able to adapt the VOR when motionmodulated prosthetic vestibular input is used. It adapts to a higher baseline stimulation, while still reacting on the dynamic component. Due to adaptation, VOR-gain, rotational axis, and symmetry increase during chronic stimulation (Merfeld et al., 2007; Gong et al., 2008; Fridman et al., 2010; Lewis et al., 2010; Dai et al., 2011a; Guyot et al., 2011b).

Knowing these adaptive capabilities of the brain opens new perspectives, not only for the engineering part (it is probably not necessary to completely mimic natural stimuli) but also for how and when to use the VI in the future. For example, patients could 
regularly turn their VI on and off in order to facilitate a more rapid adaptation and patients with Meniere's disease could use their VI as a "vestibular pacemaker" in which it is only turned on when necessary. These findings also suggest that when a vestibular prosthesis is installed, a period of adaptation is necessary in which the patients adapt to the new stimuli. Probably, a specific adaptation protocol is necessary, just like with the cochlear implant. Defining the optimal protocol will require human patient studies (Merfeld et al., 2006).

\section{FUTURE RESEARCH}

Future research should focus on engineering as well as biomechanical issues (Wall et al., 2002). At this point, engineering issues concerning power are of main concern. Therefore, only this aspect will now be discussed since other engineering issues are outside the scope of this article. Power problems can be solved by decreasing demand and increasing supply. Regarding demand, especially the sensors have a relatively high power consumption which has to be reduced. Besides, when developing these sensors, it should be taken into account that the sensors become as small as possible so they can be attached to the head. An optimal stimulus profile can also reduce power consumption by using the lowest current and frequency as possible with a power-efficient waveform (Wall et al., 2002; Macherey et al., 2006; Davidovics et al., 2011). Regarding power supply, batteries should become more powerful and there must be found a way to deal with instantaneous loss of power which could lead to dangerous situations for the patient. An external battery pack has been used previously (Merfeld et al., 2006).

\section{REFERENCES}

Agrawal, Y., Carey, J. P., Della Santina, C. C., Schubert, M. C., and Minor, L. B. (2009). Disorders of balance and vestibular function in US adults: data from the National Health and Nutrition Examination Survey, 2001-2004. Arch. Intern. Med. 169, 938-944.

Balter, S. G., Stokroos, R. J., De Jong, I., Boumans, R., Van de Laar, M., and Kingma, H. (2004). Background on methods of stimulation in galvanic-induced body sway in young healthy adults. Acta Otolaryngol. 124, 262-271.

Black, F. O., Wade, S. W., and Nashner, L. M. (1996). What is the minimal vestibular function required for compensation? Am. J. Otol. 17, 401-409.

Bonham, B. H., and Litvak, L. M. (2008). Current focusing and steering: modeling, physiology, and psychophysics. Hear. Res. 242, 141-153.

Bronte-Stewart, H. M., and Lisberger, S. G. (1994). Physiological properties of vestibular primary afferents that mediate motor learning and normal performance of the vestibulo-ocular reflex in monkeys. J. Neurosci. 14, 1290-1308.
Brummer, S. B., and Turner, M. J. (1975). Electrical stimulation of the nervous system: The principle of safe charge injection with noble metal electrodes. Bioelectrochem. Bioenerg. 2, 13-25.

Cass, S. P., Davidson, P., and Goshgarian, H. (1989). Survival of the vestibular nerve after labyrinthectomy in the cat. Otolaryngol. Head Neck Surg. 101, 459-465.

Cohen, B., Suzuki, J.-I., and Bender, M. B. (1965). Nystagmus induced ampullary nerves. Acta Otolaryngol. 422-436.

Crago, P. E., Peckham, P. H., Mortimer, J. T., Deeks, J. M., and Wouters, J. (1974). The choice of pulse duration for chronic electrical stimulation via surface, nerve, and intramuscular electrodes. Ann. Biomed. Eng. 2, 252-264. (1995). Vestibular compensation: a review of the oculomotor, neural, and clinical consequences of unilateral vestibular loss. J. Vestib. Res. 5, 67-107.

Dai, C., Fridman, G. Y., Chiang, B., Davidovics, N. S., Melvin, T. A., Cullen, K. E., and Della Santina, C. C. (2011a). Cross-axis adaptation by electrical stimulation of the

Curthoys, I. S., and Halmagyi, G. M.

Concerning biomechanical issues, determining the stimulus site in humans is the most important aspect of the biomechanical issues at this point. This can be combined by investigating the optimal stimulus profile, which could differ for each site since the distance to a nerve and current spread vary for each location. Once the prosthesis is implanted, an acclimation protocol should be established and the efficacy of the prosthesis must be evaluated by different vestibular, visual, proprioceptive, auditory, cognitive, and hearing tests. Analyzing these tests will give information about what to expect from the implant (Wall et al., 2002).

Furthermore, ethical issues should be tackled. They comprise for example safety regulations during (human) research, patient selection, alternatives for an implantable prosthesis, and costs for society.

In all aspects of the biomechanical and ethical issues, the ENT-surgeon will play an important role.

\section{CONCLUSION}

To use a basic VI in humans seems feasible in the very near future. Investigations show that electric stimulation of the canal nerves induces a nystagmus which corresponds to the plane of the canal which is innervated by the stimulated nerve branch. The brain is able to adapt to a higher baseline stimulation, while still reacting on a dynamic component. The best response will be achieved by a combination of the optimal stimulus (stimulus profile, stimulus location, precompensation), complemented by central vestibular adaptation. The degree of response will probably vary between individuals, depending on pathology and their ability to adapt.

improves 3D vestibulo-ocular reflex alignment during chronic stimulation via a head-mounted multichannel vestibular prosthesis. Exp. Brain Res. 210, 595-606.

Dai, C., Fridman, G. Y., and Della Santina, C. C. (2011b). Effects of vestibular prosthesis electrode implantation and stimulation on hearing in rhesus monkeys. Hear. Res. 277, 204-210.

Davidovics, N. S., Fridman, G. Y., Chiang, B., and Della Santina, C. C. (2011). Effects of biphasic current pulse frequency, amplitude, duration, and interphase gap on eye movement responses to prosthetic electrical stimulation of the vestibular nerve. IEEE Trans. Neural Syst. Rehabil. Eng. 19, 84-94.

Della Santina, C. C., Migliaccio, A. A., and Patel, A. H. (2007). A multichannel semicircular canal neural prosthesis using electrical stimulation to restore 3-D vestibular sensation. IEEE Trans. Biomed. Eng. 54, 1016-1030.

Dimitri, P. S., Wall, C.III, and Oas, J. G. (1996). Classification of human rotation test results using parametric modeling and multivariate statistics. Acta Otolaryngol. 116, 497-506.

Epley, J. M. (1980). Singular neurectomy: hypotympanotomy approach.
Otolaryngol. Head Neck Surg. 88, 304-309.

Feigl, G. C., Fasel, J. H., Anderhuber, F., Ulz, H., Rienmüller, R., Guyot, J. P., and Kos, I. M. (2009). Superior vestibular neurectomy: a novel transmeatal approach for a denervation of the superior and lateral semicircular canals. Otol. Neurotol. 30, 586-591.

Fridman, G. Y., Davidovics, N. S., Dai, C., Migliaccio, A. A., and Della Santina, C. C. (2010). Vestibuloocular reflex responses to a multichannel vestibular prosthesis incorporating a $3 \mathrm{D}$ coordinate transformation for correction of misalignment. J. Assoc. Res. Otolaryngol. 11, 367-381.

Frijns, J. H., de Snoo, S. L., and ten Kate, J. H. (1996). Spatial selectivity in a rotationally symmetric model of the electrically stimulated cochlea. Hear. Res. 95, 33-48.

Gacek, R. R. (1974). Transection of the posterior ampullary nerve for the relief of benign paroxysmal positional vertigo. Ann. Otol. Rhinol. Laryngol. 83, 596-605.

Gacek, R. R., and Gacek, M. R. (2002). Results of singular neurectomy in the posterior ampullary recess. $O R L$ J. Otorhinolaryngol. Relat. Spec. 64, 397-402. 
Goldberg, J. M., and Fernandez, C. (1971). Physiology of peripheral neurons innervating semicircular canals of the squirrel monkey. I. Resting discharge and response to constant angular accelerations. J. Neurophysiol. 34, 635-660.

Gong, W., Haburcakova, C., and Merfeld, D. M. (2008). Vestibulo-ocular responses evoked via bilateral electrical stimulation of the lateral semicircular canals. IEEE Trans. Biomed. Eng. 55, 2608-2619.

Gong, W., and Merfeld, D. M. (2000). Prototype neural semicircular canal prosthesis using patterned electrical stimulation. Ann. Biomed. Eng. 28, 572-581.

Gong, W., and Merfeld, D. M. (2002). System design and performance of a unilateral horizontal semicircular canal prosthesis. IEEE Trans. Biomed. Eng. 49, 175-181.

Gorman, P. H., and Mortimer, J. T. (1983). The effect of stimulus parameters on the recruitment characteristics of direct nerve stimulation. IEEE Trans. Biomed. Eng. 30, 407414.

Guyot, J. P., Sigrist, A., Pelizzone, M., Feigl, G. C., and Kos, M. I. (2011a). Eye movements in response to electrical stimulation of the lateral and superior ampullary nerves. Ann. Otol. Rhinol. Laryngol. 120, 81-87.

Guyot, J. P., Sigrist, A., Pelizzone, M., and Kos, M. I. (2011b). Adaptation to steady-state electrical stimulation of the vestibular system in humans. Ann. Otol. Rhinol. Laryngol. $120,143-149$.

Kingma, H. (2005). "Anatomie, fysiologie en onderzoek van het vestibulaire system," in Leerboek keel-, neusen oorheelkunde, ed. E. H. Huizing (Houten: Bohn Stafleu van Loghum), 115-150.

Kudo, Y., and Nomura, Y. (1996). The vestibular nerve: its course to the anterior and lateral ampullae. $O R L$ J. Otorhinolaryngol. Relat. Spec. 58, 208-212.

Lacour, M., Dutheil, S., Tighilet, B., Lopez, C., and Borel, L. (2009). Tell me your vestibular deficit, and i'll tell you how you'll compensate. Ann. N. Y. Acad. Sci. 1164, 268-278.

Leake, P. A., Hradek, G. T., and Snyder, R. L. (1999). Chronic electrical stimulation by a cochlear implant promotes survival of spiral ganglion neurons after neonatal deafness. $J$. Comp. Neurol. 412, 543-562.

Lewis, R. F., Gong, W., Ramsey, M., Minor, L., Boyle, R., and Merfeld, D. M. (2002). Vestibular adaptation studied with a prosthetic semicircular canal. J. Vestib. Res. 12, 87-94.

Lewis, R. F., Haburcakova, C., Gong, W., Makary, C., and Merfeld, D.
M. (2010). Vestibuloocular reflex adaptation investigated with chronic motion-modulated electrical stimulation of semicircular canal afferents. J. Neurophysiol. 103, 1066-1079.

Lysakowski, A. (2005). "Anatomy of the vestibular end organs and neural pathways," in Cummings Otolaryngology Head and Neck Surgery, ed. W. Cummings (Philadelphia: Elsevier), 3089-3114.

Macherey, O., van Wieringen, A., Carlyon, R. P., Deeks, J. M., and Wouters, J. (2006). Asymmetric pulses in cochlear implants: effects of pulse shape, polarity, and rate. J. Assoc. Res. Otolaryngol. 7, 253-266.

Mallinson, A. I., and Longridge, N. S. (2004). Caloric response does not decline with age. J. Vestib. Res. 14, 393-396.

McCall, A. A., Ishiyama, G. P., Lopez, I. A., Bhuta, S., Vetter, S., and Ishiyama, A. (2009). Histopathological and ultrastructural analysis of vestibular end organs in Meniere's disease reveals basement membrane pathology. BMC Ear Nose Throat Disord. 9, 4. doi: 10.1186/1472-6815-9-4

Melvill Jones, G., Guitton, D., and Berthoz, A. (1988). Changing patterns of eye-head coordination during $6 \mathrm{~h}$ of optically reversed vision. Exp. Brain Res. 69, 531-544.

Merfeld, D. M. (2004). "Vestibular prosthetics," in Neuroprosthetics: Theory and Practice, eds K. W. Horch and G. Dhillon (Singapore: World Scientific Publishing), 1115-1145.

Merfeld, D. M., Gong, W., Morrissey, J., Saginaw, M., Haburcakova, C., and Lewis, R. F. (2006). Acclimation to chronic constant-rate peripheral stimulation provided by a vestibular prosthesis. IEEE Trans. Biomed. Eng. 53, 2362-2372.

Merfeld, D. M., Haburcakova, C., Gong, W., and Lewis, R. F. (2007). Chronic vestibulo-ocular reflexes evoked by a vestibular prosthesis. IEEE Trans. Biomed. Eng. 54, 1005-1015.

Merrill, D. R., Bikson, M., and Jefferys, J. G. (2005). Electrical stimulation of excitable tissue: design of efficacious and safe protocols. J. Neurosci. Methods 141, 171-198.

Mortimer, J. (1981). "Motor prostheses," in Handbook of Physiology: The Nervous System, ed. V. B. Brooks (Bethesda, MD: American Physiological Society), 155-187.

Parnes, L. S., and McClure, J. A. (1990). Posterior semicircular canal occlusion for intractable benign paroxysmal positional vertigo. Ann. Otol. Rhinol. Laryngol. 99, 330-334.

Parnes, L. S., and McClure, J. A. (1991). Posterior semicircular canal occlusion in the normal hearing ear.
Otolaryngol. Head Neck Surg. 104, 52-57.

Rattay, F., Lutter, P., and Felix, H. (2001). A model of the electrically excited human cochlear neuron. I. Contribution of neural substructures to the generation and propagation of spikes. Hear. Res. 153, 43-63.

Rauch, S. D., Velazquez-Villasenor, L., Dimitri, P. S., and Merchant, S. N. (2001). Decreasing hair cell counts in aging humans. Ann. N. Y. Acad. Sci. 942, 220-227.

Robblee, L. S. (1990). "Electrochemical guidelines for selection of protocols and electrode materials for neural stimulation," in Neural Prostheses: Fundamental Studies, eds W. F. Agnew and D. B. McCreery (Englewood Cliffs, NJ: Prentice Hall), 2566.

Rubinstein, J. T., and Della Santina, C. C. (2002). Development of a biophysical model for vestibular prosthesis research. J. Vestib. Res. 12, 69-76.

Rubinstein, J. T., and Spelman, F. A. (1988). Analytical theory for extracellular electrical stimulation of nerve with focal electrodes. I. Passive unmyelinated axon. Biophys. J. 54, 975-981.

Sando, I., Black, F. O., and Hemenway, W. G. (1972). Spatial distribution of vestibular nerve in internal auditory canal. Ann. Otol. Rhinol. Laryngol. $81,305-314$.

Schuknecht, H. F. (1982). Behavior of the vestibular nerve following labyrinthectomy. Ann. Otol. Rhinol. Laryngol. Suppl. 97, 16-32.

Shepherd, R. K., Coco, A., and Epp, S. B. (2008). Neurotrophins and electrical stimulation for protection and repair of spiral ganglion neurons following sensorineural hearing loss. Hear. Res. 242, 100-109.

Shepherd, R. K., and Javel, E. (1999). Electrical stimulation of the auditory nerve: II. Effect of stimulus waveshape on single fibre response properties. Hear. Res. 130, 171-188.

Shepherd, R. K., Matsushima, J., Millard, R. E., and Clark, G. M. (1991). Cochlear pathology following chronic electrical stimulation using non charge balanced stimuli. Acta Otolaryngol. 111, 848-860.

Shkel, A. M., and Zeng, F. G. (2006). An electronic prosthesis mimicking the dynamic vestibular function. Audiol. Neurootol. 11, 113-122.

Suzuki, J. I., Goto, K., Tokumasu, K., and Cohen, B. (1969). Implantation of electrodes near individual vestibular nerve branches in mammals. Ann. Otol. Rhinol. Laryngol. 78 , 815-826.

Tang, S., Melvin, T. A., and Della Santina, C. C. (2009). Effects of semicircular canal electrode implantation on hearing in chinchillas. Acta Otolaryngol. 129, 481-486.

Tsuji, K., Velazquez-Villasenor, L., Rauch, S. D., Glynn, R. J., Wall, C. III, and Merchant, S. N. (2000a). Temporal bone studies of the human peripheral vestibular system. Aminoglycoside ototoxicity. Ann. Otol. Rhinol. Laryngol. Suppl. 181, 20-25.

Tsuji, K., Velazquez-Villasenor, L., Rauch, S. D., Glynn, R. J., Wall, C. III, and Merchant, S. N. (2000b). Temporal bone studies of the human peripheral vestibular system. Meniere's disease. Ann. Otol. Rhinol. Laryngol. Suppl. 181, 26-31.

Velazquez-Villasenor, L., Merchant, S. N., Tsuji K Glynn, R. J., Wall, C. III, and Rauch, S. D. (2000). Temporal bone studies of the human peripheral vestibular system. Normative Scarpa's ganglion cell data. Ann. Otol. Rhinol. Laryngol. Suppl. 181, 14-19.

Wall, C. III, Kos, M. I., and Guyot, J. P. (2007). Eye movements in response to electric stimulation of the human posterior ampullary nerve. Ann. Otol. Rhinol. Laryngol. 116, 369-374.

Wall, C. III, Merfeld, D. M., Rauch, S. D., and Black, F. O. (2002). Vestibular prostheses: the engineering and biomedical issues. J. Vestib. Res. 12, 95-113.

Zheng, C., Guyot, J. P., and Montandon, P. (1996). Ossiculoplasty by interposition of a minor columella between the tympanic membrane and stapes head. Am. J. Otol. 17, 200-202.

Conflict of Interest Statement: The authors declare that the research was conducted in the absence of any commercial or financial relationships that could be construed as a potential conflict of interest.

Received: 23 May 2011; accepted: 12 July 2011; published online: 11 August 2011. Citation: van de Berg R, Guinand N, Stokroos RJ, Guyot J-P and Kingma $H$ (2011) The vestibular implant: quo vadis? Front. Neur. 2:47. doi: 10.3389/fneur.2011.00047

This article was submitted to Frontiers in Neuro-otology, a specialty of Frontiers in Neurology.

Copyright (c) 2011 van de Berg, Guinand, Stokroos, Guyot and Kingma. This is an open-access article subject to a nonexclusive license between the authors and Frontiers Media SA, which permits use, distribution and reproduction in other forums, provided the original authors and source are credited and other Frontiers conditions are complied with. 


\section{APPENDIX}

Table A1 | Main results of vestibular implant research.

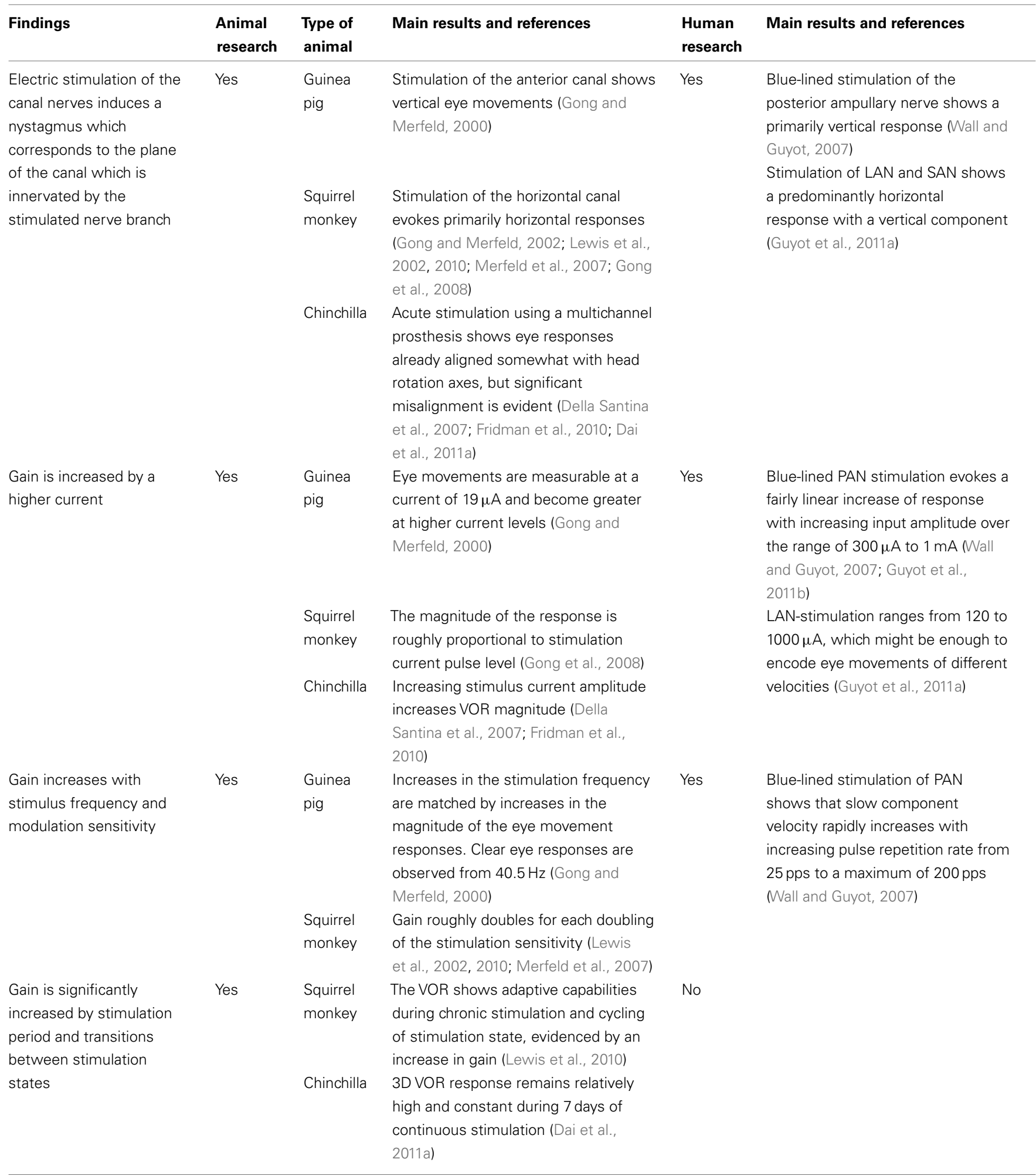


Table A1 | Continued

\begin{tabular}{|c|c|c|c|c|c|}
\hline Findings & $\begin{array}{l}\text { Animal } \\
\text { research }\end{array}$ & $\begin{array}{l}\text { Type of } \\
\text { animal }\end{array}$ & Main results and references & $\begin{array}{l}\text { Human } \\
\text { research }\end{array}$ & Main results and references \\
\hline $\begin{array}{l}\text { Bilateral stimulation } \\
\text { increases the gain }\end{array}$ & Yes & $\begin{array}{l}\text { Squirrel } \\
\text { monkey }\end{array}$ & $\begin{array}{l}\text { VOR responses evoked by bilateral } \\
\text { stimulation are the summation of the } \\
\text { responses evoked by bilateral } \\
\text { stimulation, demonstrating a gain } \\
\text { constant of } 0.24 \text { (normal }=0.26 \text {; Gong } \\
\text { et al., 2008) }\end{array}$ & No & \\
\hline & & $\begin{array}{l}\text { Squirrel } \\
\text { monkey }\end{array}$ & $\begin{array}{l}\text { The nystagmus evoked by baseline } \\
\text { stimulation disappears within } 6 \mathrm{~h} \text { to } \\
1 \text { day of the chronic stimulation turned } \\
\text { on (Lewis et al., 2002, 2010; Merfeld } \\
\text { et al., 2007) }\end{array}$ & & $\begin{array}{l}\text { When continuous electrical } \\
\text { stimulation at } 400 \mu \mathrm{A} \text { is turned on } \\
\text { for the first time, strong nystagmic } \\
\text { beats are almost absent from } \\
\text { recordings after } 27 \text { min (Guyot et al., } \\
2011 \text { b) }\end{array}$ \\
\hline $\begin{array}{l}\text { The vestibular system } \\
\text { adapts to static baseline } \\
\text { stimulation, but not to } \\
\text { dynamic modulation }\end{array}$ & Yes & $\begin{array}{l}\text { Squirrel } \\
\text { monkey }\end{array}$ & $\begin{array}{l}\text { Sinusoidally modulated stimulation } \\
\text { yields a sinusoidally modulated VOR, } \\
\text { even after acclimation to the baseline } \\
\text { stimulation (Merfeld et al., 2006) } \\
\text { Animals show horizontal VORs for } \\
\text { periods exceeding } 90 \text { days when } \\
\text { pulse-rate is modulated, while } \\
\text { nystagmus evoked by baseline } \\
\text { stimulation has disappeared (Merfeld } \\
\text { et al., 2007; Lewis et al., 2010) }\end{array}$ & Yes & $\begin{array}{l}\text { Once a patient is in an adapted } \\
\text { state, it is possible to elicit smooth } \\
\text { oscillatory eye movements by } \\
\text { modulating the amplitude or } \\
\text { frequency of the stimulation (Guyot } \\
\text { et al., } 2011 \mathrm{~b} \text { ) }\end{array}$ \\
\hline \multirow[t]{2}{*}{$\begin{array}{l}\text { Cross-axis adaptation is } \\
\text { possible in the vestibular } \\
\text { system }\end{array}$} & Yes & $\begin{array}{l}\text { Squirrel } \\
\text { monkey }\end{array}$ & $\begin{array}{l}\text { A horizontal VOR can develop even if } \\
\text { the stimulated posterior canal is } \\
\text { orthogonal to the velocity sensor of } \\
\text { the prosthesis (Lewis et al., 2002) }\end{array}$ & No & \\
\hline & & Chinchilla & $\begin{array}{l}\text { Cross-axis adaptation considerably } \\
\text { improves 3D VOR alignment during the } \\
\text { first week of chronic stimulation (Dai } \\
\text { et al., 2011a) }\end{array}$ & & \\
\hline $\begin{array}{l}\text { Time constant of the } \\
\text { evoked VOR is smaller } \\
\text { than the time constant of } \\
\text { the prosthesis }\end{array}$ & Yes & $\begin{array}{l}\text { Squirrel } \\
\text { monkey }\end{array}$ & $\begin{array}{l}\text { The time constant of the VOR response } \\
\text { was smaller than the time constant of } \\
\text { the prosthesis (Merfeld et al., 2007; } \\
\text { Gong et al., 2008; Lewis et al., 2010) }\end{array}$ & No & \\
\hline
\end{tabular}


Table A1 | Continued

Findings
Improvement in
VOR-symmetry is still
uncertain

Misalignment of VOR-axis improves significantly during prosthetic use

$\begin{array}{ll}\begin{array}{l}\text { Animal } \\ \text { research }\end{array} & \begin{array}{l}\text { Type of } \\ \text { animal }\end{array} \\ \text { Yes } & \text { Squirrel } \\ & \text { monkey }\end{array}$

\section{Main results and references}

During the first 2 weeks of stimulation,

there is a decline in difference between

the ipsi- and contralateral gains of

$71-78 \%$ when stimulated in the low

sensitivity mode (Lewis et al., 2010)

Chinchilla VOR-asymmetry did not change

significantly during 1 week of

prosthetic use (Dai et al., 2011a)

Squirrel During chronic stimulation, the initial

monkey VOR-axis $\left(45^{\circ}-56^{\circ}\right)$ is shifted in the

plane closer to the compensatory

orientation of $90^{\circ}\left(73^{\circ}-83.5^{\circ}\right.$; Lewis

et al., 2010)

Chinchilla Seven days of continuous prosthetic

use shows a significant improvement

in VOR alignment (Dai et al., 2011a)

\section{Human Main results and references \\ research}

No

No 